\title{
A novel computational strategy for DNA methylation imputation using mixture regression model (MRM)
}

\author{
Fangtang Yu, Chao Xu, Hong-Wen Deng and Hui Shen *
}

\author{
*Correspondence: \\ hshen3@tulane.edu \\ Center for Bioinformatics \\ and Genomics, Department \\ of Biostatistics and Data \\ Science, School of Public \\ Health and Tropical Medicine, \\ Tulane University, New \\ Orleans, LA 70112, USA
}

\begin{abstract}
Background: DNA methylation is an important heritable epigenetic mark that plays a crucial role in transcriptional regulation and the pathogenesis of various human disorders. The commonly used DNA methylation measurement approaches, e.g., Illumina Infinium HumanMethylation-27 and -450 BeadChip arrays (27 K and $450 \mathrm{~K}$ arrays) and reduced representation bisulfite sequencing (RRBS), only cover a small proportion of the total CpG sites in the human genome, which considerably limited the scope of the DNA methylation analysis in those studies.

Results: We proposed a new computational strategy to impute the methylation value at the unmeasured $\mathrm{CpG}$ sites using the mixture of regression model (MRM) of radial basis functions, integrating information of neighboring $\mathrm{CpG}$ and the similarities in local methylation patterns across subjects and across multiple genomic regions. Our method achieved a better imputation accuracy over a set of competing methods on both simulated and empirical data, particularly when the missing rate is high. By applying MRM to an RRBS dataset from subjects with low versus high bone mineral density (BMD), we recovered methylation values of $\sim 300 \mathrm{~K} \mathrm{CpGs}$ in the promoter regions of chromosome 17 and identified some novel differentially methylated CpGs that are significantly associated with BMD.
\end{abstract}

Conclusions: Our method is well applicable to the numerous methylation studies. By expanding the coverage of the methylation dataset to unmeasured sites, it can significantly enhance the discovery of novel differential methylation signals and thus reveal the mechanisms underlying various human disorders/traits.

Keywords: Methylation, Imputation, Mixture of regression models, Epigenomic association studies

\section{Background}

DNA methylation is one of the most important epigenetic marks in the human genome, during which a methyl group $\left(-\mathrm{CH}_{3}\right)$ is added to the $\mathrm{C}-5$ position of a cytosine of DNA. In mammals, more than $98 \%$ of DNA methylation occurs in the context of neighboring cytosine and guanine nucleotides (CpGs) in somatic cells, while as much as a quarter of all methylation appears in a non-CpG context in embryonic stem cells (ESCs) [1, 2]. author(s) and the source, provide a link to the Creative Commons licence, and indicate if changes were made. The images or other third party material in this article are included in the article's Creative Commons licence, unless indicated otherwise in a credit line to the material. If material is not included in the article's Creative Commons licence and your intended use is not permitted by statutory regulation or exceeds the permitted use, you will need to obtain permission directly from the copyright holder. To view a copy of this licence, visit http:// creativecommons.org/licenses/by/4.0/. The Creative Commons Public Domain Dedication waiver (http://creativecommons.org/publi cdomain/zero/1.0/) applies to the data made available in this article, unless otherwise stated in a credit line to the data. 
DNA methylation plays a crucial role in transcriptional regulation. Typically, the occurrence of methylation in the promoter region of a gene will suppress the transcription of the gene, while the occurrence of methylation in the gene bodies is commonly associated with transcriptional activation [3, 4]. The significance of DNA methylation mediated epigenetic regulation has been well established by biological functional studies on embryonic development, genomic imprinting, X-chromosome inactivation, and the pathogenesis of various human disorders [5].

Recent advances in high-throughput microarray and sequencing techniques have made it possible to measure DNA methylation level on a genome-wide scale in a large number of samples thus facilitate association studies of DNA methylation with the phenotype of interest, i.e., EWAS (epigenome-wide association study) [6]. By performing EWAS, researchers have identified differentially methylated CpGs (DMCs), regions (DMRs), or genes associated with various diseases, including cancer [7], Alzheimer's disease [8], rheumatoid arthritis [9], and diabetes [10]. The gold standard for DNA methylation measurement is whole-genome bisulfite sequencing (WGBS), which can comprehensively quantify 26 million of the 28 million CpGs in the human genome [11]. However, it is still cost-prohibitive to apply WGBS to large-scale studies [12]. On the other hand, the commonly used cost-effective DNA methylation measurement methods only cover a small portion of the human genome. For example, the Illumina Infinium HumanMethylation-27 and -450 BeadChip arrays (27 K and $450 \mathrm{~K}$ arrays) and reduced representation bisulfite sequencing (RRBS) method only measure approximately $0.1,2$, and $8-10 \%$ of the total CPG sites, respectively [11]. The low genome coverage of methylation data from these techniques considerably limited the scope of the DNA methylation analysis in those studies.

To effectively boost the power of detecting DMCs/DMRs in DNA methylation studies using low coverage methylation assays, several recent studies have proposed a variety of computational approaches [13-18] for in silico prediction of DNA methylation values at unmeasured CpG sites. Some of these DNA methylation imputation approaches used classical statistical/machine learning methods such as ordinary linear regression, logistic regression, random forest, and support vector machine (SVM) approaches [14, 15], while others have adopted more advanced methods, including functional regression (13), deep learning [16], and gradient boosting [17]. Although these methods have some success in imputing the methylation value, there are also some limitations and weaknesses, especially in the utilization of different kinds of information for imputation. If we assume a data matrix of DNA methylation value with rows representing subjects and columns representing CPG sites, the information used for imputation of missing values of a data matrix can be classified into three categories: (1) external annotation information, (2) correlations between subject/samples (rows), and (3) correlations between neighboring CpG sites (columns). The annotation information used in the existing methods often included co-localization of the CPG sites with DNA sequence annotations (e.g. promoters, gene body, CpG islands), cis-regulatory elements (e.g., DNase I hypersensitive sites, specific transcription factor binding sites, and histone modification marks), as well as DNA sequence properties (e.g. GC content, integrated haplotype scores) [19]. Some imputation methods [14-17] use a large number of annotation information as input features for methylation imputation. However, these features are identical for each subject 
and thus are not informative for predicting subject-specific DNA methylation patterns. Also, for some tissue/cell types, many of these annotation features are currently not available. Several imputation studies using the correlation between samples as prediction features required measurement of DNA levels available using different platforms or from different sets of samples and established site-specific prediction model only on the overlapped CpG sites in the two different data types. For example, Zhang et al. developed a prediction model for predicting methylation level of $450 \mathrm{~K}$-specific probes using the probe shared between the two arrays as predictors, trained and tested on DNA methylation data of tumor tissues from 194 patients measured by both $27 \mathrm{~K}$ and $450 \mathrm{~K}$ array [13]. Ma et al. [18] assumed that locus-specific methylation differences between tissues are highly consistent across individuals and they built linear regression and SVM model to predict locus-specific methylation in the target tissue based on methylation in the surrogate tissue. It remains unclear in those studies if the established model could be applied directly to an external dataset to predict methylation levels. Thus, the practical value of using only the correlation between samples for methylation imputation is vague: if different data types (e.g. DNA methylation data measured in different platforms or tissues) are already available for the same set of subjects, there's no need for researchers to impute one data type by the other one. Furthermore, the information of neighboring CpGs was highly trivialized in previous methods. For instance, several methods only took an average of the methylation level of one upstream and downstream CpGs weighted by the genomic distance [15], or simply drop those features in the regression model, without taking into account of the whole methylation profile in a genomic region [14]. Fan's method also assessed the correlation of flanking CpGs in a panel of surrogate tissue and selected the WGBS methylation value from the tissue which has the most similar local methylation pattern as imputation score for the target locus in a target tissue [15], then combined with the weighted sum of methylation levels of the closest CpGs. Although this method integrates the correlation between samples and correlation between neighboring $\mathrm{CpGs}$, it lacks mathematical rigor. Since the local methylation profile is the only subject-specific information among the three kinds of information aforementioned, it should be modeled and integrated with other types of information more carefully to get subject-specific imputation of methylation values. For downstream association analysis in EWAS, only on those CpGs sites with enough variance in methylation values across subjects are informative.

Taking the limitations in the previous methylation imputation methods into account, we developed a novel computational strategy to impute the methylation value at the unmeasured CPG site in the low coverage DNA methylation data. We hypothesized that the linear model of radial basis functions (RBFs) can be used to capture the information of the local methylation profile, and we proposed a mixture regression model (MRM) of RBFs to impute the methylation values in a genomic region for multiple subjects simultaneously. The regional modeling is based on recent findings that DNA methylation has a similar correlation pattern to that of linkage disequilibrium (LD) in genetic SNP variation $[20,21]$. Based on the existence of such a correlation structure of neighboring CpG sites in DNA methylation data, the RBFs were used to fit the curve of methylation profile in predefined regions while the MRM can cluster the multiple methylation profiles simultaneously. We fit the MRM in two steps, first across subjects and then across 
regions (Fig. 1), then ensembled the two imputation values for each missing CpG site with stacked regression. By this approach, the MRM of RBFs can effectively impute the methylation values at the missing CPG sites by taking advantage of the information of neighboring CpGs, the similarities of local methylation patterns not only across subjects but also across multiple genomic regions within each subject.

The rest of the article is organized as follows. In "Results" section, we described the simulation scheme for evaluating the performance of MRM and presented the performance of MRM in both simulated and real methylation data comparing with several other commonly used imputation methods. As an empirical demonstration, we also applied the MRM method to an RRBS methylation dataset for an association study of bone mineral density (BMD). In "Discussion" section, we elaborated on the strength, limitations, and some future extensions of our study. In "Methods" section, we introduced in detail the statistical model of the proposed MRM method.

\section{Results}

To imputing the methylation values, we first built a regional model, which is independent for each predefined genomic region across subjects, assuming similarity in local methylation profiles across subjects. Then we built a subject model in different genomic regions of each subject to get another imputation of the methylation value at the missing points (Fig. 1, details see "Methods"). The final imputation value is the stacked value

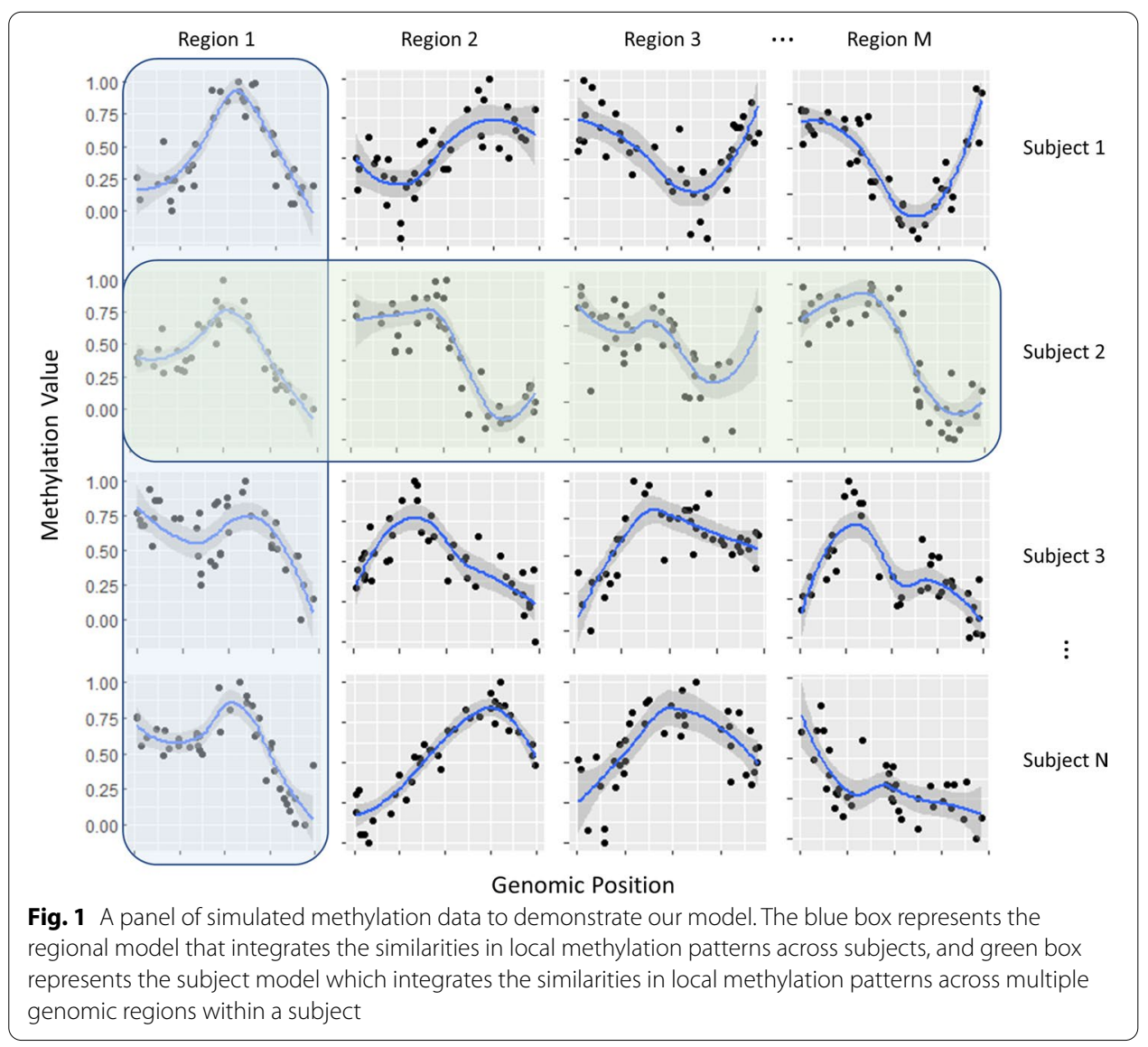


of imputation values from both the regional and the subject model. The performance of our method was benchmarked on both simulated and empirical data.

\section{Benchmark the imputation performance on simulated data}

We simulated DNA methylation data of multiple regions based on linear models of RBFs. For each region, the subjects were randomly assigned to different clusters. The MRM along with the other four DNA methylation imputation methods were applied to the simulated data, and their performance was evaluated under different conditions with varying noise levels, sample sizes, and missing rates.

\section{Simulation scheme}

\section{Simulation by MRM model}

To evaluate the imputation performance of the MRM, we simulated methylation of 100 independent regions each with $50 \mathrm{CpG}$ sites. In each region, the methylation values were simulated directly by a mixture regression model of RBFs. Four sub-population clusters were generated with the following cluster proportions: $\pi=10 \%, 20 \%, 30 \%$, and $40 \%$. In total, we simulated 12 datasets with varying sample size, noise level, and missing rate to evaluate the performance of MRM in different settings (Additiaonal file 1: Table S1). The sample size was set to be $20,50,100$, or 200 , which is typical in current WGBS studies [22]. To mimic the inherent noise, Gaussian noise with mean zero and varying variance was introduced to the probability of methylated CpG sites. The missing rate, which represents the percentage of CpGs in a region that is not measured, was set to be $20-80 \%$. The wide range of missing rates could represent either the missing rate within the WGBS platform for different subjects or the missing rate in other lower coverage platforms compared with WGBS [11].

\section{Simulation by profile-based bisulfite sequencing data simulator}

We used the pWGBSSimla software [23] to simulate WGBS data that is more similar to real data. This algorithm calculated the smoothed methylation rates based on the real cell-type-specific methylation profiles. WGBS data in a $100 \mathrm{~kb}$ region in chromosome 1 of mesenchymal stem cells of sample size 20 and 100 were simulated, resulting in a total of $850 \mathrm{CpGs}$ per sample. The CpGs were distributed into 17 continuous regions each containing 50 CpGs. We randomly deleted $20-80 \%$ CpGs to generate artificial missing values. MRM models were trained by the remaining CpGs to predict the artificial missing values. The center parameter $\mu_{j}$ was set to be equally distributed along the region and the number of RBF centers was set to be 50 for each region. The positions were scaled between -1 and 1 and the scale parameter $\gamma$ of RBF was set to be -10 .

\section{Competing methods}

To benchmark the imputation performance of MRM, we compared it with four other imputation strategies that have been proposed and applied in previous studies. First, as a baseline approach, we computed the weighted sum of methylation values of the closest upstream and downstream CpG sites. The weight was inversely proportional to the distance from the target $\mathrm{CpG}$ [15]. Using the four features (methylation value and distance of the closest upstream and downstream CpG sites), we trained a random forest (RF) 
model on each region. This was essentially the same method as proposed in [14], but without adding any external annotation information, in order to ensure that information used for imputation by different methods only comes from methylation data itself so that the comparison was fair. We also applied the k-nearest neighbors (KNN) algorithm, which has been widely used for imputing missing values in gene expression data [24] and also been proposed for imputing DNA methylation data by [14]. As practiced previously $[24,25]$, the input of the $\mathrm{KNN}$ algorithm is a matrix of methylation values with CpG sites in the rows and subjects in the columns. For each CpG site with missing values, the $\mathrm{KNN}$ algorithm finds the $\mathrm{k}$ nearest neighbors (CpGs) using a Euclidean metric between the columns for which that $\mathrm{CpG}$ is not missing and all the columns of each other CpGs in the genomic region, and impute the missing elements by averaging those non-missing elements of its neighbors. If all the neighbors are missing in a particular CpGs, the algorithm will use the regional column mean for that CpG. Finally, we compared our method to a recently developed method, Melissa (MEthyLation Inference for Single cell Analysis), a Bayesian hierarchical method to cluster cells based on local methylation patterns [26]. At each genomic region, Melissa imputation performs clustering for local methylation profiles, which is similar to our model. However, the Melissa model get the cluster membership by clustering the whole methylome of cells instead of clustering in each region independently. As designed for single cell bisulfite sequencing data, the input need to be binary values ( 0 or 1$)$ indicating the methylation status and the output is a continuous value from 0 to 1 . To make the comparion feasible, we binarized the methylation values using cutoff 0.5 to generate the input for Melissa model.

\section{Effect of noise level}

To evaluate the robustness of imputation methods to noise, we simulated five datasets by adding different levels of Gaussian noise with varying standard deviations from 0.1 to 0.9 . The sample size was set to be 20 and the missing rate to be $20 \%$ in all datasets. Figure 2 shows the comparison of different imputation methods with varying noise levels. The performance of all algorithms decreased with the increasing noise level, in terms of significant decrease of correlation, increase of RMSE, and decrease of AUC (test statistics in Additiaonal file 1: Table S2). However, the MRM method consistently outperforms all the other tested methods, and the performance exceedance of MRM over other methods also increases with increasing noise levels.






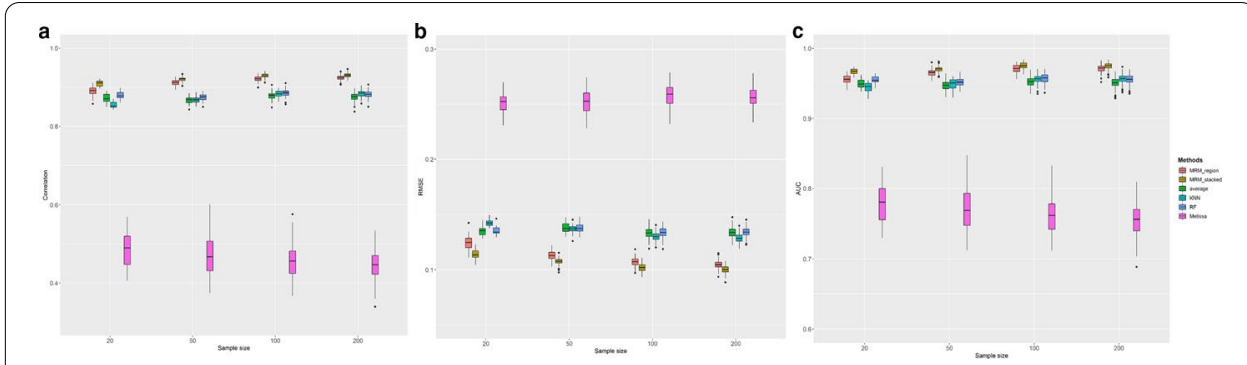

Fig. 3 Box plot of a correlation, b RMSE, c AUC of six imputation algorithm on simulated data under the effect of sample size. Each colored boxplot indicates the imputation performance on the given number of subjects. Abbreviations see legend of Fig. 2

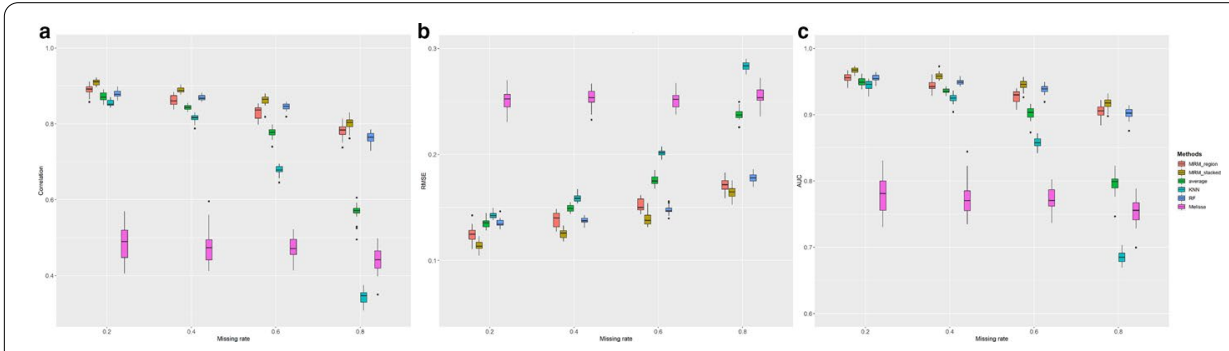

Fig. 4 Box plot of a correlation, b RMSE, c AUC of six imputation algorithm on simulated data under the effect of the missing rate. Each colored boxplot indicates the imputation performance on 20 subjects. Abbreviations see legend of Fig. 2

\section{Effect of sample size}

Imputation accuracy may be influenced by the sample size of the dataset. A smaller sample size will lead to increases in the variance of imputed values [27]. For algorithms that use many features, the design matrix tends to be ill-conditioned if the sample size is limited. To evaluate the effect of sample size on the performance of our imputation method, we simulated four datasets with sample size 20,50, 100, and 200. The standard deviation of Gaussian noise was set to be 0.2 and the missing rate was fixed to be $20 \%$ at random. As shown in Fig. 3, our method significantly outperformed other methods in terms of correlation, RMSE, and AUC at all sample sizes (test statistics in Additiaonal file 1: Table S3). For most algorithms, the variance in correlation and RMSE are generally increasing with the decrease of sample size. However, the changes of correlation and RMSE of our method with varying sample sizes were less dramatic, highlighting the performance stability of MRM. On the other hand, the performance of RF and Melissa methods even declined with the increasing sample size, suggesting that larger sample size might not always be beneficial for those imputation frameworks that using global features if the methylation data only have regional cluster patterns.

\section{Effect of data missing rate}

We tested the DNA methylation imputation methods on four simulated data sets with different levels of missing rate $(20 \%, 40 \%, 60 \%$, and $80 \%)$. Like the comparisons for other conditions, our MRM methods significantly outperform other tested methods in terms 
of correlation, RMSE, and AUC, under each missing rate level (Fig. 4, test statistics in Additiaonal file 1: Table S4). As expected, we observed significant decrease of correlation, increase of RMSE, and decrease of AUC with increasing missing rate for all tested methods, particularly for algorithms using simple local features, e.g. KNN and up/downstream weighted sum algorithm. However, the decrease of imputation performance of our MRM method is much less dramatic than the other methods, demonstrating the exceptional robustness of our methods even when the missing rate is high. This might be due to the utilization of complementary information across subjects, neighboring $\mathrm{CpG}$ sites, and other genomic regions.

We also compared the imputation performance on pWGBSSimla simulated data with different levels of missing rate $(20 \%, 40 \%, 60 \%$, and $80 \%)$ at sample size 20 and 100 . Although the performance of all methods on pWGBSSimla data was relatively low, the MRM methods still significantly outperform other tested methods under both sample size settings (Additiaonal file 1: Figure S1, Table S5).

\section{Benchmark the imputation performance on real methylation data Dataset}

We downloaded a WGBS dataset measured from subcutaneous adipose tissue (SAT) of 19 subjects in the Multiple Tissue Human Expression Resource (MuTHER) cohort from the ArrayExpress database [access ID: E-MTAB-3549]. The subjects were Caucasian females aged between 40 and 87 years old. The WGBS data measured $\sim 27$ million CpG sites, with mean genome coverage 6.3-fold (1.0-12.9) and CpG-discovery saturated at sixfold coverage [28].

\section{Data preprocess}

For demonstration purposes, we only applied the imputation methods to chromosome 18. We clustered the CpG sites on this chromosome into regions based on their physical distance and the similarity of methylation values of two $\mathrm{CpG}$ sites by an $\mathrm{R}$ package Aclust [29]. Specifically, the algorithm first scanned all the CpG sites in WGBS dataset to identify pairs of CpG sites for which the physical distance between the two CpG sites is smaller than $3000 \mathrm{bp}$ and the Pearson correlation of the methylation values at the two sites is greater than 0.3, then the two CpG sites with all the sites wedged in between them were merged into one region. Only two adjacent regions can be merged at each iterative step. The similarity of methylation values in two regions was defined using the average correlation between all sites in the two regions.

We mapped the CpG sites to promoter regions of up to $\pm 5 \mathrm{~kb}$ around the transcription start site (TSS) of UCSC genes. The promoter region containing more than 50 measured CpG sites were then filtered by variance, skewness [30] and runs test for nonrandomness [31]. The promoter region was kept for imputation only if the methylation data in that region was available for more than 15 subjects, with variance $>0.1$, skewness $>-1$, and the number of runs $<15$. As many CpG sites are either completely methylated or unmethylated across individuals in a genomic region [18] the filtering criteria were applied to make sure the pattern of methylation profile in the selected region follows the assumption of the mixture of regression models. After applying the clustering and filtering, a total of 47 regions with more than $50 \mathrm{CpGs}$ were selected. And we define 
this scenario as Condition 0 . To imitate the conditions of various missing rates, we randomly deleted the measured DNA methylation values at $20 \%, 40 \%, 60 \%$, and $80 \%$ of CpG sites in the selected regions of each subject to generate artificial missing values.

While the clustering and filtering procedures aimed to ensure the existence of LD-like patterns [20] in the selected genomic regions and to make the assumptions of MRM satisfied, we further investigate the performance of MRM in the following two more general conditions in which the assumption of MRM might be violated: Condition $1 \mathrm{We}$ randomly selected 2500 continuous CpGs without clustering or filtering and divided them into 50 sliding windows with $50 \mathrm{CpG}$; Condition 2 We randomly selected 50 promoter regions and pruned them to regions that contain 50 CpGs (25 CpGs upstream and 25 CpGs downstream the TSS), without performing clustering and filtering.

\section{Imputation}

After generating the artificial missing values, the methylation data of the remaining CpGs were used to train the MRMs. The center parameter $\mu_{j}$ and scale parameter of the RBFs was set to be the same as the pWGBSSimla simulated data (see "Simulation by profile-based bisulfite sequencing data simulator" in "Result"). We applied our proposed method to impute the missing values at the target $\mathrm{CpG}$ sites based on the regional model only and the stacked model (weighted sum of the regional and the subject model). The other four methods were also used to impute these missing values and then compared with our methods under different settings of missing rates.

\section{Performance on real data}

The comparison of correlation, RMSE, and AUC between our methods and other methods for imputing the DNA methylation values at the targeted CpG sites were shown in Fig. 5. Consistent with the simulation results, we observed decreased correlation, increased RMSE, and decreased AUC with an increasing missing rate for all methods. At the lower missing rate $(r=0.2$ and 0.4$)$, MRM exhibited comparable performance with KNN and outperforms all the other tested imputation methods. However, the correlation drops dramatically for KNN and up/downstream weighted sum methods, similar to the simulation results. MRM also outperformed other methods at higher missing rates $(r=0.6$ and 0.8$)$, highlighting the strength of MRM for taking advantage of information from multiple sources. It is noteworthy that the Melissa algorithm performs relatively

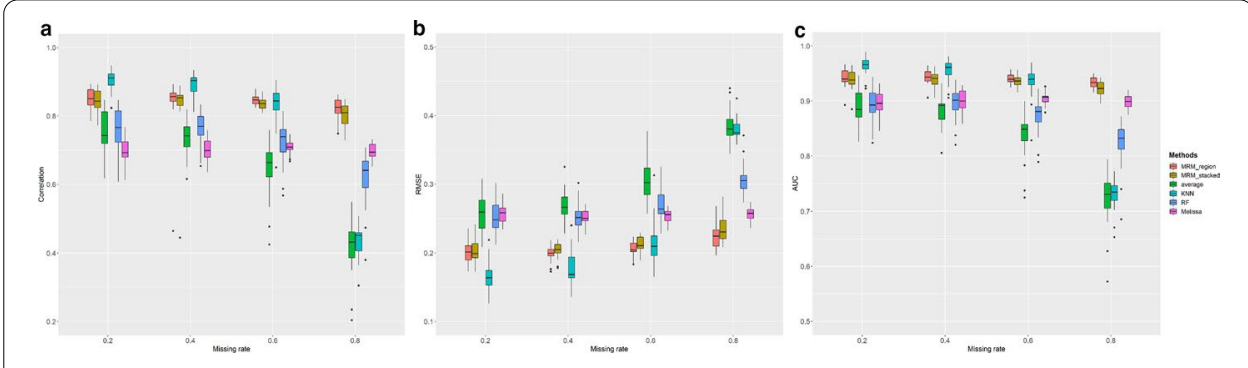

Fig. 5 Box plot of a correlation, b RMSE, c AUC of six imputation algorithms on real WGBS data under the effect of the missing rate. Each colored boxplot indicates the imputation performance on 19 subjects. Abbreviations see legend of Fig. 2 
stable under the influence of the missing rate compared with other methods. Additionally, MRM using only the regional model performs slightly better than using the stacked regional and subject model, indicating that the local methylation patterns across multiple genomic regions within a subject introduced more noise than useful information for methylation imputation. The performance of MRM under conditions 1 and 2 is similar to that under condition 0 (Additiaonal file 1: Figures S2, S3). However, the performance of MRM at the same missing rate across different conditions are significantly different (Additiaonal file 1: Table S6). The MRM achieves the best performance in regions selected with clustering and filtering (condition 0 ) among the three conditions. Although its performance in regions defined by the sliding window approach (condition 1), which ignored the correlation pattern in methylation data, is inferior to the other two conditions, the MRM methods still outperformed other methods at a high missing rate. This result also emphasized that the regional correlation pattern is important for MRM to make accurate imputation.

\section{Application to an epigenomic association study}

After benchmarking the imputation accuracy of MRM, we further demonstrated the empirical implementation of our method by applying the MRM to an RRBS dataset from our recent epigenomic association study of BMD [32]. In this study, DNA methylation profiles of peripheral blood monocytes (PBM) were determined by RRBS in 118 Caucasian females, including 64 subjects with high hip BMD (Z-scores $\geq 0.8)$ and 54 subjects with low hip BMD (Z-scores $\leq-0.8$ ). The BMD Z-score was defined as the number of standard deviations a subject's BMD differed from the mean BMD of their age-, gender-, and ethnicity-matched population. We are particularly interested in imputing methylation values on chromosome 17 because recent studies have found several genes on chromosome 17 were associated with BMD [33] and the methylation level of a set of CpGs on chromosome 17 mediated the association between SNP and BMD [34]. MRM was applied to promoter regions ( $\pm 5 \mathrm{~kb}$ around TSS) where more than $50 \mathrm{CpGs}$ were measured in each region taking all the subjects into account. To increase the computational speed, only the regional model is applied. We used methylKit [35] to identify DMCs between low BMD and high BMD group, adjusting for age, body mass index (BMI), drinking status, smoking status, and 1st principal component (PC) of methylation. Before imputation, only CpG sites with $\geq$ threefold coverage in at least 30 subjects in each BMD group were included in the association analysis, while after imputation, all the $\mathrm{CpG}$ sites in the processed promoter regions were included. CpGs with significant difference in methylation levels (Bonferroni adjusted $\mathrm{p}$-value $<0.05$ ) between the two BMD groups were defined as DMCs. The DMCs were annotated to the genes corresponding to the promoter region. The Genomic Regions Enrichment of Annotations Tool (GREAT) v3.0.0 [36] was used to evaluate whether the nearby genes of DMCs are enriched in any gene and human phenotypes ontology terms.

We limited our analyses to 35,713 CpGs in 1,369 qualified promoter regions on chromosome 17 for demonstration purposes. Without imputation, we only identified 348 DMCs with p-value $<1.49 \mathrm{E}-6(0.05 / 35,713)$ significantly associated with BMD. In contrast, by applying the MRM algorithm, we imputed methylation values for 309,165 CpGs in the tested promoter regions and identified 2459 significant DMCs with 
Table 1 Mouse phenotype enrichments for BMD-associated DMCs

\begin{tabular}{llc}
\hline Term name & Fold enrichment & FDR Q-Val \\
\hline Brachyphalangia & 21.48521 & $3.95 \mathrm{E}-33$ \\
Short metacarpal bones & 21.48521 & $3.95 \mathrm{E}-33$ \\
Short metatarsal bones & 21.48521 & $3.95 \mathrm{E}-33$ \\
Decreased trabecular bone mass & 21.48521 & $3.95 \mathrm{E}-33$ \\
Decreased trabecular bone connectivity density & 9.427838 & $3.95 \mathrm{E}-21$ \\
\hline
\end{tabular}

Table 2 DMC enriched genes that were associated with BMD or bone metabolism

\begin{tabular}{lllll}
\hline Gene & $\begin{array}{l}\text { Number of imputed } \\
\text { DMCs }\end{array}$ & Traits & GwasCatlog Study ID & PMID \\
\hline SPECC1 & 16 & Heel BMD & GCST006433 & 30048462 \\
SPATA20 & 23 & Heel BMD & GCST007066 & 30595370 \\
USP36 & 11 & Heel BMD & GCST006979 \\
SMG6 & 13 & Heel BMD & GCST006979 & 30598549 \\
& 13 & Heel BMD & GCST006433 & 30598549 \\
MYO1D & 12 & Osteoclast differen- & NA & 30048462 \\
& & tiation & NA & 21567867 \\
ASB16-AS1 & 12 & BMD & & 29763751 \\
\hline
\end{tabular}

p-value $<1.62 \mathrm{E}-7(0.05 / 309,165)$, among which 2452 were new DMCs. The DMCs were distributed in 594 unique promoter regions (median number of DMCs in a promoter region $=2$ ). The GREAT analysis showed the nearby genes of the imputed DMCs were significantly enriched in some BMD related terms of mouse phenotype (Table 1). We further checked the functions of the 42 genes whose promoter regions contained more than 10 imputed DMCs. Interestingly, six genes (SPECC1, SPATA20, USP36, SMG6, $M Y O 1 D$, and ASB16-AS1), have been found to be associated with BMD or bone-related phenotypes in previous studies [37-43] (Table 2, Additiaonal file 1: Table S7). These results indicated that our algorithm can successfully impute the methylation values at the unmeasured CpG sites and enhance the power to identify novel DMCs in epigenomic association studies.

\section{Discussion}

We proposed a novel computational strategy, MRM of RBFs, to impute the missing or unmeasured $\mathrm{CpG}$ methylation values by effectively integrating three kinds of information: the information of neighboring CpGs, the similarities in local methylation patterns across subjects, and the similarities in local methylation patterns across multiple genomic regions within a subject, thus addressed the low coverage problem of the methylation data generated in many cost-efficient platforms. The MRM method can be used to impute methylation values in pre-defined genomic regions, for example, the promoter region or to impute methylation values on the genome-wide scale using sliding windows. The real data benchmarking results of MRM performance under several preprocessing conditions indicated that this method is more suitable for imputing missing values on a regional basis, especially when the regions have an LD-like correlation pattern. In this study, we only implement the MRM on selected genome regions for demonstration 
purposes. The computation time of MRM is practicable for implementing on a whole genome scale, although the computation time is longer compared with other methods under different sample sizes for imputing simulated data (Additiaonal file 1: Table S8). Considering the excellence in performance at a high missing rate, it worth trading a reasonable increase amount of computation time for the imputation accuracy. The MRM imputation does not require any external information, such as regulatory annotation and DNA sequence properties. It is straight forward to fit the output of MRM as a new local feature into some existing methylation imputation frameworks as [15] and [14].

Based on our simulation result, the MRM method could recover the WGBS data (correlation $=0.8$ ) after deleting $80 \%$ of data points. This missing rate could represent the difference in genome coverage between WBGS and RRBS. Thus, our method could be applied to most of the existing RRBS data to expand the coverage and achieve better imputation accuracy. However, genome coverage of microarray-based methods are even lower, analogous to WGBS data with a missing rate $>97 \%$. It will be unrealistic to recover whole-genome methylation data with that high missing rate without reference or external information. We would suggest not use MRM to impute microarray data only. If WGBS data from the same cell type were available, users could combine methylation data from different platforms (i.e. WGBS and microarray) as input, and the higher coverage methylation data will perform as a reference for imputation.

Despite the advantages of the MRM algorithm, we noticed that it is important to evaluate the discrepancies between the real methylation data and the statistical model we proposed. As opposed to the simulated data, the subject model provided more noise than useful information in the real data and thus the stacked model performed less well than the regional model. This may due to the fact that the selected regions in our study do not share common patterns across the genome, which conflicts assumption of the subject MRM model. We believe that common patterns exist across certain genome regions, e.g. promoters of genes in the same pathway or regulatory network. To ensure the stacked model works better, the users may need to incorporate some prior annotation information in selecting specific regions they want to impute. Otherwise, we recommend using the regional model in general.

Some other future directions of the MRM algorithm are worth exploring. First, since MRM is a finite mixture regression model, the number of clusters has to be specified. It is computationally burdensome to fit multiple MRMs and do model selection based on the model likelihood. Instead of doing the model selection from several models with different cluster number, we recommend users choose a reasonably larger cluster number. As we found in the simulation study that when the number of clusters is set to be larger than the real number of clusters, the performance of MRM as good as if the number of clusters is correctly specified (Additiaonal file 1: Figure S4). An alternative scheme would assume a mixture model with an unknown number of components and the most common choice of the prior distributions for this clustering problem is the Dirichlet process (DP) [44]. The DP mixture model has been successfully used to perform clustering of gene expression data by microarray $[45,46]$, and could be extended to DNA methylation analysis in future studies. Besides, the association analysis after imputation is conducted throughout a large number of $\mathrm{CpG}$ sites, which will lead to severe multiple testing problems. It has been well recognized that DMRs might have more prominent 
biological significance compared with single CpG sites [47]. Therefore, we will attempt to integrate the MRM imputation algorithm with region-based differential methylation analysis approaches $[21,48-50]$ to develop novel computation tools that could simultaneously do DNA methylation imputation and region-based association testing.

\section{Conclusions}

The proposed MRM method provided a state-of-art performance for methylation data imputation. On both simulated and empirical DNA methylation data, the MRM method achieved a better imputation performance over a set of competing methods, particularly when the missing rate is high. By applying the proposed method to an in-house DNA methylation for osteoporosis, we identified some novel differential methylation signals that are significantly associated with BMD and demonstrated that this method is well applicable to existing methylation studies that were conducted with commonly used, low genome coverage methylation analysis platforms and is expected to significantly enhance the discovery of novel DNA methylation regulated genes and mechanisms underlying various human disorders/traits.

\section{Methods}

\section{Statistical modeling of the methylation profile}

As in many previous practical studies, we are interested in imputing the methylation level of a CpG site as a ratio ranging from 0 to 1 , where 0 represents no methylation and 1 represents $100 \%$ methylation at a CpG site. We assumed that the methylation data of the $m$-th genomic region in the $n$-th subject is represented by a vector $y_{m n}$ of length $I$, where $\mathrm{m}=1,2 \ldots, \mathrm{M}$ and $\mathrm{n}=1,2, \ldots, \mathrm{N}$.

\section{Regional model}

A subject-specific model was developed for methylation profiles in each predefined region. For each region $m$, we assumed that the methylation profiles of the $N$ subjects can be partitioned into at most $K$ clusters. Suppose there are $I$ measured CpG sites. For one subject, let $\boldsymbol{x}=\left(x_{1}, x_{2}, \ldots x_{I}\right)$ be a vector of the CpG locations; $f(\boldsymbol{x})$ be a function representing the methylation profile. We assumed $f(\boldsymbol{x})$ is a linear combination of a set of radial basis functions $h_{j}()$ of the input space $\boldsymbol{x}$

$$
\mathrm{y}=f(\boldsymbol{x}, \boldsymbol{w})=\sum_{j=0}^{J} w_{j} h_{j}(\boldsymbol{x})=H \boldsymbol{w}
$$

where $H$ is an $I \times J$ design matrix with element $h_{i j}=\exp \left(-\delta|| x_{i}-\mu_{j} \|^{2}\right) ; x_{i}$ are the component of vector $\boldsymbol{x} ; \mu_{j}$ are the selected centers of the basis functions; $\delta$ is the scale parameter; $\boldsymbol{w}=\left(w_{1}, \ldots, w_{J}\right)^{T} ; \mu_{j}$ represents the RBF centers; $J$ represents the number of RBF centers.

Parameters $\boldsymbol{w}$ in model [1] can be obtained simply by solving the normal equation

$$
\left(H^{\prime} H\right) \hat{\boldsymbol{w}}=H^{\prime} \boldsymbol{y}
$$

For $N$ subjects from $K$ clusters i.e. regression with $K$ specific set of regression coefficients, the probability that subject $n$ belong to cluster $k$ is $\pi_{k}$. The methylation profile of subject $n$ region $m$ can be written as 


$$
y_{i n}=f(\boldsymbol{x}, \boldsymbol{w})=\sum_{j=0}^{J} w_{k j m n} \exp \left(-\delta\left\|x_{i}-\mu_{j}\right\|^{2}\right) \text { if } \quad z_{k n}=1
$$

where $z_{n k}$ is a latent indicator variable of whether subject $n$ belongs to cluster $k$, $z_{n k} \in\{0,1\}$ and $p\left(z_{k n}=1\right)=\pi_{k}$.

The observed methylation data $(\boldsymbol{x}, \boldsymbol{y})$ can be viewed as data repeatedly measured from $N$ subjects, each has $I$ observations. Thus, the conditional log-likelihood of $Y$ can be written as a weighted sum of $K$ Gaussian distributions.

$$
L(\boldsymbol{y} \mid \boldsymbol{x})=\sum_{n=1}^{N} \sum_{i=1}^{I} \log \sum_{k=1}^{K} \pi_{k} N\left(H\left(x_{i n}\right) w_{k} \mid \Sigma\right)
$$

To impute the missing values, we first need to learn the model parameters of each cluster and the posterior probability that a subject belongs to cluster $k$ using the available data in a genomic region of all subjects. Then, given the genomic position of the missing point, the methylation value can be computed based on Eq. (2). The regression coefficient $w_{k}$ can be learned by maximizing Eq. (3). But the $H$ matrix could become high dimensional as the number of RBFs increases and the number of observed CpG sites remains fixed. To ameliorate this issue, we maximize a penalized version of Eq. (3), by adding an $l 1$ regularization term to the log-likelihood function which will encourage the weights to decay to zero as proposed by Stadler [51].

$$
L_{p e n}(y \mid x)=\sum_{n=1}^{N} \sum_{i=1}^{I} \log \sum_{k=1}^{K} \pi_{k} N\left(H\left(x_{i n}\right) w_{k} \mid \Sigma\right)+\lambda \sum_{k=1}^{K} \pi_{k}\left\|w_{k}\right\|_{1}
$$

The parameter set $\theta=\left\{\pi_{k}, \boldsymbol{w}_{\boldsymbol{k}}, \Sigma\right\}$ was estimated by maximizing the penalized likelihood function using the EM algorithm using functions in R package FlexMix [52].

Since we don't know the true clustering of subjects, we fit the MRMs with different numbers of clusters $(K)$. The maximum number is set to be proportional to the sample size $N$. The model with K clusters that yields the lowest ICL (Integrated completed likelihood) was retained, which has been proven to be a very popular approach to choose the number of clusters in model-based clustering [53]. The penalty parameter $\lambda$ is chosen in grid search by a tenfold cross validation.

\section{Subject model and stacking}

While the regional model assumes similarity in local methylation profiles across subjects, some studies also found that the methylation profile in proximal regions with similar annotation properties may share the same patterns [13, 14]. Thus, we fit the MRM model in different genomic regions of each subject to get another imputation of the methylation value at the missing points (Fig. 1). The model fitting process is analogous to the regional model.

To integrate multiple imputation models, we used a least-square regression model to combine the outputs from the regional and subject models. This stacking approach forms linear combinations of different predictors at each locus to improve the prediction accuracy [54].

$$
\boldsymbol{\beta}=\operatorname{argmin}\left[y-\left(\beta_{r} \widehat{y_{r}}+\beta_{s} \widehat{y_{s}}\right)\right] \text { s.t. } \beta_{r}, \beta_{s}>0
$$


where $\widehat{y_{r}}$ and $\widehat{y_{s}}$ are imputed methylation values from the regional and the subject models, respectively. The non-negative regularization has been shown to produce stacking predictors with substantially reduced prediction errors [54]. To train the stacking regression model, we adopted bootstrapping to randomly generate artificial missing values at the locations which were not overlapped with true missing locations. The weights were estimated by Eq. (5) based on the imputed and true values on the artificial missing points. The averaged value of each weight on 100 times bootstrapping was used for prediction.

\section{Evaluation metric}

The performance was evaluated by the root mean squared error (RMSE) and the correlation between the true and imputed values, which are defined as the following:

$$
\begin{aligned}
& \text { RMSE }=\sqrt{\frac{\sum_{m=1}^{M} \sum_{i=1}^{I_{m}}\left(y_{i m}-\widehat{y_{i m}}\right)^{2}}{M I}} \\
& \text { correlation }=\frac{\sum_{m=1}^{M} \sum_{i=1}^{I_{m}}\left(y_{i m}-\overline{y_{i m}}\right)\left(\widehat{y_{i m}}-\widehat{y_{i m}}\right)}{\sqrt{\sum_{m=1}^{M} \sum_{i=1}^{I_{m}}\left(y_{i m}-\overline{y_{i m}}\right)^{2}} \sqrt{\sum_{m=1}^{M} \sum_{i=1}^{I_{m}}\left(\widehat{y_{i m}}-\widehat{y_{i m}}\right)^{2}}}
\end{aligned}
$$

where $y_{i m}$ and $\widehat{y_{i m}}$ are the true and imputed value of the $i$-th missing point in the $m$ -th region of the genome in a subject, respectively, $I_{m}$ is the number of missing points in region $m$ and $M$ is the number of genomic regions. In addition, to make fair comparisons with Melissa which only takes binary methylation values as input, the area under the receiver operating characteristic curve (AUC) of the true and predicted values with a cut-off of 0.5 was also calculated for all the tested imputation methods. We performed t-tests to compare the AUC, correlation, and RMSE under different settings to determine whether observed differences between the performance were statistically significant.

\section{Supplementary information}

Supplementary information accompanies this paper at https://doi.org/10.1186/s12859-020-03865-z.

Additional file 1. Supplementary materials (Supplementary Tables S1-S8, Supplementary Figures S1-S4).

\section{Abbreviations}

AUC: Area under the receiver operating characteristic curve; BMD: Bone mineral density; CpG: Cytosine and guanine dinucleotides; DMC: Differentially methylated CpG; DMR: Differentially methylated region; DP: Dirichlet process; ESCs: Embryonic stem cells; EWAS: Epigenome-wide association study; GREAT: Genomic Regions Enrichment of Annotations Tool; KNN: K-nearest neighbors; LD: Linkage disequilibrium; Melissa: Methylation inference for single-cell analysis; MRM: Mixture of regression models; MUTHER: Multiple Tissue Human Expression Resource; RBF: Radial basis functions; RF: Random forest; RMS: Eroot mean squared error; RRBS: Reduced representation bisulfite sequencing; SAT: Subcutaneous adipose tissue; SVM: Support vector machine; TSS: Transcription start site; WGBS: Whole-genome bisulfite sequencing.

\section{Acknowledgements}

This research was supported in part using high-performance computing (HPC) resources and services provided by Technology Services at Tulane University, New Orleans, LA.

\section{Authors' contributions}

FY and HS conceived the study. FY designed the statistical methods. FY and CX processed and analyzed the data. HS and HWD contributed to manuscript preparation and feedback. All authors read and approved the manuscript. 


\section{Funding}

This study was partially supported or benefited by grants from the National Institutes of Health [R01AR069055,

R01MH104680, R01AR059781, R01AG061917, U19AG055373, and P20GM109036], and the Edward G. Schlieder Endowment and the Drs. W. C. Tsai and P.T. Kung Professorship in Biostatistics from Tulane University. The funders had no role in study design, data collection and analysis, decision to publish, or preparation of the manuscript.

\section{Availability of data and materials}

The source code is available at https://github.com/yuft2003/MRM.The WGBS datasets for benchmarking are available in the ArrayExpress database (access ID: E-MTAB-3549) and RRBS datasets of osteoporosis are available in dbGaP database (access ID: phs001960.v1.p1).

\section{Ethics approval and consent to participate}

Not applicable.

\section{Consent for publication}

Not applicable.

\section{Competing interests}

The authors declare that they have no competing interests.

Received: 8 February 2020 Accepted: 9 November 2020

Published online: 01 December 2020

\section{References}

1. Jaenisch R, Bird A. Epigenetic regulation of gene expression: how the genome integrates intrinsic and environmental signals. Nat Genet. 2003;33:245-54.

2. Jin B, Li Y, Robertson KD. DNA methylation: superior or subordinate in the epigenetic hierarchy? Genes Cancer. 2011:2(6):607-17.

3. Laurent L, Wong E, Li G, Huynh T, Tsirigos A, Ong CT, et al. Dynamic changes in the human methylome during differentiation. Genome Res. 2010;20(3):320-31.

4. Lister R, Pelizzola M, Dowen RH, Hawkins RD, Hon G, Tonti-Filippini J, et al. Human DNA methylomes at base resolution show widespread epigenomic differences. Nature. 2009:462(7271):315-22.

5. Jones PA. Functions of DNA methylation: islands, start sites, gene bodies and beyond. Nat Rev Genet. 2012:13(7):484-92.

6. Bergman Y, Cedar H. DNA methylation dynamics in health and disease. Nat Struct Mol Biol. 2013;20(3):274-81.

7. Kleinman CL, Gerges N, Papillon-Cavanagh S, Sin-Chan P, Pramatarova A, Quang D-AK, et al. Fusion of TTYH1 with the C19MC microRNA cluster drives expression of a brain-specific DNMT3B isoform in the embryonal brain tumor ETMR. Nat Genetics. 2014;46(1):39-44.

8. De Jager PL, Srivastava G, Lunnon K, Burgess J, Schalkwyk LC, Yu L, et al. Alzheimer's disease: early alterations in brain DNA methylation at ANK1, BIN1, RHBDF2 and other loci. Nat Neurosci. 2014;17(9):1156-63.

9. Liu Y, Aryee MJ, Padyukov L, Fallin MD, Hesselberg E, Runarsson A, et al. Epigenome-wide association data implicate DNA methylation as an intermediary of genetic risk in rheumatoid arthritis. Nat Biotechnol. 2013;31(2):142-7.

10. Soriano-Tárraga C, Jiménez-Conde J, Giralt-Steinhauer E, Mola-Caminal M, Vivanco-Hidalgo RM, Ois A, et al. Epigenome-wide association study identifies TXNIP gene associated with type 2 diabetes mellitus and sustained hyperglycemia. Hum Mol Genet. 2015;25(3):609-19.

11. Sun Z, Cunningham J, Slager S, Kocher J-P. Base resolution methylome profiling: considerations in platform selection, data preprocessing and analysis. Epigenomics. 2015;7(5):813-28.

12. Zhou L, Ng HK, Drautz-Moses DI, Schuster SC, Beck S, Kim C, et al. Systematic evaluation of library preparation methods and sequencing platforms for high-throughput whole genome bisulfite sequencing. Sci Rep. 2019;9(1):1-16.

13. Zhang G, Huang KC, Xu Z, Tzeng JY, Conneely KN, Guan W, et al. Across-platform imputation of DNA methylation levels incorporating nonlocal information using penalized functional regression. Genet Epidemiol. 2016;40(4):333-40.

14. Zhang W, Spector TD, Deloukas P, Bell JT, Engelhardt BE. Predicting genome-wide DNA methylation using methylation marks, genomic position, and DNA regulatory elements. Genome Biol. 2015;16(1):14.

15. Fan $\mathrm{S}, \mathrm{Li}$ C, Ai R, Wang M, Firestein GS, Wang W. Computationally expanding infinium HumanMethylation450 BeadChip array data to reveal distinct DNA methylation patterns of rheumatoid arthritis. Bioinformatics. 2016:32(12):1773-8

16. Angermueller C, Lee HJ, Reik W, Stegle O. Erratum to: DeepCpG: accurate prediction of single-cell DNA methylation states using deep learning. Genome Biol. 2017;18(1):90

17. Zou LS, Erdos MR, Taylor DL, Chines PS, Varshney A, McDonnell Genome I, et al. BoostMe accurately predicts DNA methylation values in whole-genome bisulfite sequencing of multiple human tissues. BMC Genomics. 2018;19(1):390.

18. Ma B, Wilker EH, Willis-Owen SA, Byun H-M, Wong KC, Motta V, et al. Predicting DNA methylation level across human tissues. Nucleic Acids Res. 2014:42(6):3515-28.

19. Voight BF, Kudaravalli S, Wen X, Pritchard JK. A map of recent positive selection in the human genome. PLoS Biol. 2006;4(3):e72

20. Liu Y, Li X, Aryee MJ, Ekström TJ, Padyukov L, Klareskog L, et al. GeMes, clusters of DNA methylation under genetic control, can inform genetic and epigenetic analysis of disease. Am J Hum Genet. 2014;94(4):485-95.

21. Hansen KD, Langmead B, Irizarry RA. BSmooth: from whole genome bisulfite sequencing reads to differentially methylated regions. Genome Biol. 2012;13(10):R83. 
22. Nair SS, Luu P-L, Qu W, Maddugoda M, Huschtscha L, Reddel R, et al. Guidelines for whole genome bisulphite sequencing of intact and FFPET DNA on the Illumina HiSeq XTen. Epigenetics Chromatin. 2018;1 1(1):24.

23. Chung R-H, Kang C-Y. pWGBSSimla: a profile-based whole-genome bisulfite sequencing data simulator incorporating methylation QTLs, allele-specific methylations and differentially methylated regions. Bioinformatics. 2020;36(3):660-5.

24. Hastie T, Tibshirani R, Sherlock G, Eisen M, Brown P, Botstein D. Imputing missing data for gene expression arrays. 1999.

25. Troyanskaya O, Cantor M, Sherlock G, Brown P, Hastie T, Tibshirani R, et al. Missing value estimation methods for DNA microarrays. Bioinformatics. 2001;17(6):520-5.

26. Kapourani C-A, Sanguinetti G. Melissa: Bayesian clustering and imputation of single-cell methylomes. Genome Biol. 2019:20(1):61

27. Lin D, Zhang J, Li J, Xu C, Deng H-W, Wang Y-P. An integrative imputation method based on multi-omics datasets. BMC Bioinformatics. 2016;17(1):247.

28. Busche S, Shao X, Caron M, Kwan T, Allum F, Cheung WA, et al. Population whole-genome bisulfite sequencing across two tissues highlights the environment as the principal source of human methylome variation. Genome Biol. 2015;16(1):290.

29. Sofer T, Schifano ED, Hoppin JA, Hou L, Baccarelli AA. A-clustering: a novel method for the detection of co-regulated methylation regions, and regions associated with exposure. Bioinformatics. 2013;29(22):2884-91.

30. Groeneveld RA, Meeden G. Measuring skewness and kurtosis. J R Stat Soc Ser D (Statistician). 1984;33(4):391-9.

31. Barton DE, David FN. Multiple, RUNS. Biometrika. 1957;44(1-2):168-78.

32. Qiu C, Yu F, Su K, Zhao Q, Zhang L, Xu C, Hu W, Wang Z, Zhao L, Tian Q, Wang Y. Multi-omics data integration for identifying osteoporosis biomarkers and their biological interaction and causal mechanisms. Iscience. 2020;23(2):100847.

33. Mo X-B, Lu X, Zhang Y-H, Zhang Z-L, Deng F-Y, Lei S-F. Gene-based association analysis identified novel genes associated with bone mineral density. PLOS ONE. 2015;10(3):e0121811.

34. Yu F, Qiu C, Xu C, Tian Q, Zhao L-J, Wu L, et al. Mendelian randomization identifies CpG methylation sites with mediation effects for genetic influences on BMD in peripheral blood monocytes. Front Genetics. 2020;11:60.

35. Akalin A, Kormaksson M, Li S, Garrett-Bakelman FE, Figueroa ME, Melnick A, et al. methylKit: a comprehensive R package for the analysis of genome-wide DNA methylation profiles. Genome Biol. 2012;13(10):R87.

36. McLean CY, Bristor D, Hiller M, Clarke SL, Schaar BT, Lowe CB, et al. GREAT improves functional interpretation of cisregulatory regions. Nat Biotechnol. 2010;28(5):495.

37. Birnbaum MJ, Picco J, Clements M, Witwicka H, Yang M, Hoey MT, et al. Using osteoclast differentiation as a model for gene discovery in an undergraduate cell biology laboratory. Biochem Mol Biol Educ. 2010;38(6):385-92.

38. Kichaev G, Bhatia G, Loh P-R, Gazal S, Burch K, Freund MK, et al. Leveraging polygenic functional enrichment to improve GWAS power. Am J Hum Genetics. 2019a;104(1):65-75.

39. Kim SK. Identification of 613 new loci associated with heel bone mineral density and a polygenic risk score for bone mineral density, osteoporosis and fracture. PLoS ONE. 2018;13(7):e0200785.

40. Meng X-H, Chen X-D, Greenbaum J, Zeng Q, You S-L, Xiao H-M, et al. Integration of summary data from GWAS and eQTL studies identified novel causal BMD genes with functional predictions. Bone. 2018;113:41-8.

41. Morris JA, Kemp JP, Youlten SE, Laurent L, Logan JG, Chai RC, et al. An atlas of genetic influences on osteoporosis in humans and mice. Nat Genet. 2019;51(2):258,

42. Galea GL, Meakin LB, Williams CM, Hulin-Curtis SL, Lanyon LE, Poole AW, et al. Protein kinase Ca (PKCa) regulates bone architecture and osteoblast activity. J Biol Chem. 2014;289(37):25509-22.

43. Kemp JP, Morris JA, Medina-Gomez C, Forgetta V, Warrington NM, Youlten SE, et al. Identification of 153 new loci associated with heel bone mineral density and functional involvement of GPC6 in osteoporosis. Nat Genet. 2017:49(10):1468-75.

44. Neal RM. Markov chain sampling methods for Dirichlet process mixture models. J Comput Graph Stat. 2000;9(2):249-65.

45. Park J-H, Kyung M. Bayesian curve fitting and clustering with Dirichlet process mixture models for microarray data. J Korean Stat Soc. 2019:48(2):207-20.

46. Sun J, Herazo-Maya JD, Kaminski N, Zhao H, Warren JL. A Dirichlet process mixture model for clustering longitudinal gene expression data. Stat Med. 2017;36(22):3495-506.

47. Eckhardt F, Lewin J, Cortese R, Rakyan VK, Attwood J, Burger M, et al. DNA methylation profiling of human chromosomes 6, 20 and 22. Nat Genet. 2006:38(12):1378.

48. Aryee MJ, Jaffe AE, Corrada-Bravo H, Ladd-Acosta C, Feinberg AP, Hansen KD, et al. Minfi: a flexible and comprehensive Bioconductor package for the analysis of Infinium DNA methylation microarrays. Bioinformatics. 2014;30(10):1363-9.

49. Jaffe AE, Murakami P, Lee H, Leek JT, Fallin MD, Feinberg AP, et al. Bump hunting to identify differentially methylated regions in epigenetic epidemiology studies. Int J Epidemiol. 2012;41(1):200-9.

50. Peters TJ, Buckley MJ, Statham AL, Pidsley R, Samaras K, Lord RV, et al. De novo identification of differentially methylated regions in the human genome. Epigenetics Chromatin. 2015;8(1):6.

51. Städler N, BühImann P, Van De Geer S. l 1-penalization for mixture regression models. Test. 2010;19(2):209-56.

52. Leisch F. Flexmix: A general framework for finite mixture models and latent glass regression in R. 2004.

53. Bertoletti $M$, Friel N, Rastelli R. Choosing the number of clusters in a finite mixture model using an exact integrated completed likelihood criterion. Metron. 2015;73(2):177-99.

54. Breiman L. Stacked regressions. Mach Learn. 1996;24(1):49-64.

\section{Publisher's Note}

Springer Nature remains neutral with regard to jurisdictional claims in published maps and institutional affiliations. 